\title{
HELMINTIASIS GASTROINTESTINAL EN CUYES (Cavia porcellus) DE GRANJAS DE CRIANZA FAMILIAR-COMERCIAL ENANCASH, PERÚ
}

\author{
Gastrointestinal Helminthiasis in Guinea Pig (Cavia porcellus) Family- \\ Commercial Breeding Farms in Ancash, Peru
}

\author{
Cristina García J. ${ }^{1}$, Amanda Chávez V. ${ }^{1,3}$, Rosa Pinedo V. ${ }^{1}$, Francisco Suárez A. ${ }^{2}$
}

\section{Resumen}

El objetivo del estudio fue determinar la prevalencia, identificación y cuantificación de helmintos presentes en cuyes (Cavia porcellus) en la fase de acabado de granjas de crianza familiar-comercial, en la zona de Caraz, Ancash. Se recolectó el estómago, intestino delgado e intestino grueso de 100 cuyes y se identificaron y contabilizaron los parásitos presentes. Se encontró una prevalencia de $89 \%$ de nemátodos gastrointestinales. Los parásitos identificados fueron Paraspidodera uncinata, Trichuris spp, Capillaria spp y Trichostrongylus colubriformis con prevalencias de 83, 31, 18 y 2\%, respectivamente. No se encontró diferencias significativas entre sexos. Asimismo, la frecuencia de monoparasitismo, biparasitismo y triparasitismo fue de 49, 35 y $5 \%$, respectivamente.

Palabras clave: cuy, nemátodos, crianza familiar-comercial, Caraz, Ancash

\section{Abstract}

The objective of this study was to determine the prevalence, identification and quantification of helminths present in guinea pigs (Cavia porcellus) in the finishing phase from family-commercial breeding farms in the district of Caraz, Ancash, Peru. The stomach, small intestine and large intestine of 100 animals were collected and all parasites were identified and counted. The prevalence of gastrointestinal nematodes was $89 \%$. Identified parasites were Paraspidodera uncinata, Trichuris spp, Capillaria spp and Trichostrongylus colubriformis showing prevalence of $83,31,18$ and $2 \%$ respectively and without differences due to sex. The frequency of monoparasitism, biparasitism and triparasitism was 49,35 and 5\% respectively.

Key words: guinea pig, nematodes, family-commercial breeding, Caraz, Ancash

\footnotetext{
${ }^{1}$ Laboratorio de Parasitología y Microbiología, ${ }^{2}$ Laboratorio de Medicina Veterinaria Preventiva, Facultad de Medicina Veterinaria, Universidad Nacional Mayor de San Marcos, Lima

${ }^{3}$ E-mail: achavezvg@gmail.com
}

Recibido: 21 de enero de 2013

Aceptado para publicación: 20 de abril de 2013 


\section{INTRODUCCIÓN}

El cuy, cobayo o curí (Cavia porcellus) es un mamífero roedor originario de la zona andina de Bolivia, Colombia, Ecuador y Perú. La mayor población de cuyes se encuentra en el Perú, registrándose una producción anual de 16500 toneladas de carne proveniente de cuyes criados básicamente en sistemas de producción familiar (Chauca, 1997).

Las enfermedades parasitarias son las responsables de más de las dos terceras partes de la morbilidad y mortalidad en esta especie. Estas enfermedades se caracterizan por ser de manifestación lenta, pasando desapercibidas por los criadores, produciendo retraso en el crecimiento y una mayor susceptibilidad para contraer otro tipo de enfermedades que conllevan a producir pérdidas económicas no cuantificadas por los criadores (INIA, 1994; Florián, 1999).

Existen diversos factores epidemiológicos que contribuyen a la presencia de parásitos gastrointestinales, tales como deficientes condiciones higiénico sanitarias, sobrepoblación animal, crianza promiscua con otras especies domésticas, y ausencia de programas de control y prevención; además, se debe considerar los bajos niveles socioeconómicos y culturales del poblador andino (INIA, 1994). Asimismo, la alimentación que reciben los cuyes de crianza familiar-comercial es en base a residuos de cosechas, concentrado y pastos cultivados, que muchas veces son compartidos con el ganado existente en la zona, lo que posibilitaría la infección cruzada con algunas formas parasitarias que afectan a los rumiantes.

Existe poca información en el país sobre la identificación de parásitos internos que afectan al cuy, sobre todo en zonas alejadas de la capital, lo cual dificulta la elaboración de programas de desparasitación. Los objetivos del estudio fueron determinar la prevalencia, identificación y cuantificación de helmintos presentes en cuyes en la fase de acabado de granjas de crianza familiar-comercial en el distrito de Caraz, Ancash.

\section{Materiales y Métodos}

\section{Lugar de Estudio}

El estudio se realizó en el distrito de Caraz, provincia de Huaylas, departamento de Ancash, Perú, ubicado a $2256 \mathrm{msnm}$. La zona tiene una clima seco en otoño, invierno y primavera y es templado húmedo en verano, con temperatura anual entre 16 y $25^{\circ} \mathrm{C}$. La época de lluvia es entre diciembre a abril y la época seca entre mayo a noviembre (SENAMHI, 2009; INEI, 2012).

El muestreo se realizó entre junio a setiembre de 2011, en cuyes adultos de saca de granjas de crianza familiar-comercial, donde fueron alimentados a base de forraje y concentrado. Las muestras fueron recolectadas al momento del sacrificio del animal.

\section{Tamaño Muestral}

Al carecer de información sobre la frecuencia de helmintos en cuyes del lugar, se realizó un muestreo piloto en seis granjas familiares comerciales de cuatro centros poblados del distrito. Se colectaron muestras de 30 cuyes adultos, escogidos al azar por los criadores, encontrándose una prevalencia de 93\%. Esta se utilizó en la fórmula para estimar una proporción basada en la aproximación normal a la distribución binomial, con 95\% de confianza y $5 \%$ de precisión (Daniel, 1996), resultando un tamaño muestral de 100 animales.

\section{Recolección y Análisis de Muestras}

Los cuyes fueron sacrificados como parte del plan de saca de los productores en las instalaciones de crianza o en los lugares de venta. Se recolectó el estómago e intestino delgado y grueso de cada animal, ligando cada extremo para evitar el derrame del con- 
Cuadro 1. Prevalencia de nematodos, según especie, y carga parasitaria ${ }^{1}$ en 100 cuyes machos y hembras en la fase de acabado en el distrito de Caraz, Ancash (2011)

\begin{tabular}{|c|c|c|c|c|c|}
\hline \multirow{2}{*}{ Especie parasitaria } & \multicolumn{2}{|c|}{ Cuyes } & \multirow{2}{*}{$\begin{array}{l}\text { Prevalencia } \\
(\%)\end{array}$} & \multirow{2}{*}{$\begin{array}{c}\text { Carga } \\
\text { parasitaria } \\
\text { (promedio) }\end{array}$} & \multirow{2}{*}{$\begin{array}{c}\text { Valor } \\
\text { máximo } \\
\text { (n) }\end{array}$} \\
\hline & Machos & Hembras & & & \\
\hline $\begin{array}{l}\text { Paraspidodera } \\
\text { uncinata }\end{array}$ & 49 & 34 & 83 & 13.7 & 106 \\
\hline Trichuris spp & 17 & 14 & 31 & 4.2 & 38 \\
\hline Capillaria spp & 8 & 10 & 18 & 22.8 & 232 \\
\hline $\begin{array}{l}\text { Trichostrongylus } \\
\text { colubriformis }\end{array}$ & 2 & 0 & 2 & 1 & 2 \\
\hline
\end{tabular}

${ }^{1}$ Número de parásitos

Cuadro 2. Asociación parasitaria en 89 cuyes (macho y hembra) positivos a nematodos en el distrito de Caraz, Ancash (2011)

\begin{tabular}{|c|c|c|}
\hline Asociación parasitaria & $\begin{array}{c}\text { Cuyes } \\
(\%)\end{array}$ & Especies asociadas \\
\hline \multirow[t]{3}{*}{ Monoparasitismo } & 55.1 & P. uncinata \\
\hline & & Trichuris spp \\
\hline & & Capillaria spp \\
\hline \multirow[t]{4}{*}{ Biparasitismo } & 39.3 & P. uncinata + Trichuris spp \\
\hline & & P. uncinata + Capillaria $\mathrm{spp}$ \\
\hline & & P. uncinata $+T$. colubriformis \\
\hline & & Trichuris sp.+ Capillaria spp \\
\hline Triparasitismo & 5.6 & P. uncinata + Trichuris $\mathrm{spp}+$ Capillaria $\mathrm{spp}$ \\
\hline
\end{tabular}

tenido, y se colocaron en bolsas plásticas o en frascos de boca ancha. Las muestras fueron conservadas con fijador a base de alcohol-formol-ácido acético (AFA), preparado según Botero y Restrepo (2006).

Se recolectó la totalidad de parásitos gastrointestinales presentes en los órganos colectados. Se utilizó la solución de lactofenol para la clarificación de los helmintos (Castro y Guerrero, 2006). La identificación y clasificación de los parásitos se basó en las características morfológicas (Skrjabin et al., 1954; Yamaguti, 1959; Soulsby, 1987; Vicente et al., 1997). Posteriormente, se realizó el montaje según Hakaro y Cadral (1998). 


\section{Análisis de Datos}

Se determinó el número promedio de parásitos por animal (carga parasitaria) y la prevalencia de la parasitosis, considerándose como positiva aquella muestra que contenga al menos un helminto (Thrusfield, 1990; Wisnivesky, 2003).

La prevalencia de la parasitosis se expresó en forma porcentual con sus respectivos intervalos de confianza al 95\%. La posible asociación entre la variable sexo con la presencia de especies parasitarias se analizó mediante la prueba de Chi cuadrado. Se utilizó el paquete estadístico STATA® v. 10.0.

\section{Resultados}

La prevalencia de parásitos gastrointestinales fue de $89 \pm 6.1 \%$, siendo $85.7 \%$ en hembras y $91.4 \%$ en machos aunque sin diferencia estadística. Asimismo, se encontraron cuatro especies de nematodos (Cuadro 1), donde la especie de mayor prevalencia fue Paraspidodera uncinata $(83 \%)$.

Se determinaron las asociaciones parasitarias (Cuadro 2), encontrándose $55.1 \%$ de cuyes positivos a nematodos con una especie de parásito (monoparasitismo), siendo la especie más prevalente $P$. uncinata. Asimismo, se encontró un 39.3\% de biparasitismo, siendo la asociación más frecuente $P$. uncinata y Trichuris spp. Solo el 5.6\% de los animales presentaron tres especies parasitarias ( $P$. uncinata, Trichuris spp y Capillaria spp).

\section{Discusión}

El $89 \%$ de prevalencia de parasitismo gastrointestinal encontrado en los cuyes correspondió a nematodos, toda vez que no se encontraron cestodos (Cuadro 1). Resultados en estudios similares en cuyes sacrifica- dos en un mercado de Lima (Ruiz, 1961) y en cuyes provenientes de crianza familiar en Cajamarca (Merino, 1991) presentaron prevalencias de nematodos del 81 y $96 \%$, respectivamente; confirmando que esta especie, bajo las condiciones de crianza local, se encuentra severamente parasitada.

Paraspidodera uncinata, el parásito de mayor frecuencia en los cuyes en estudio, tiene una amplia distribución en América del Sur y su presencia ha sido descrita en varias familias de roedores (Griffiths, 1971) sin mostrar una merma en su comportamiento productivo (Dean y Stephen, 2007). Sin embargo, en infecciones altas se observan signos clínicos como pérdida de peso, debilidad y diarrea (Wescott, 1976).

No hay estudios que correlacionen la carga parasitaria de nematodos en cuyes domésticos con los niveles de parasitismo alto, moderado y leve establecido en rumiantes; sin embargo, y pese a las elevadas cargas parasitarias de Capillaria spp (22.8 parásitos) y P. uncinata (13.7 parásitos) observados en el presente estudio (Cuadro 1), los animales mostraban un buen estado de carnes y se encontraban aparentemente sanos. Por otro lado, es posible que los resultados obtenidos sean inferiores a los que mostrarían animales jóvenes, debido a que estos son más susceptibles a las parasitosis (Florián, 1999; Cordero del Campillo et al., 2001).

Se ha demostrado que $P$. uncinata parasita a cuyes silvestres (Cavia aperea) sobre los $4000 \mathrm{msnm}$ con prevalencias de $72 \%$ en la zona de Cusco (Tió, 1970) y de 28 a $42 \%$ en diversas partes de la sierra peruana (Dittmar, 2002); asimismo, en Brasil se encontraron prevalencias de $87.8 \%$ (Pereira, 2006). Estudios de prevalencia de $P$. uncinata en el país en cuyes domésticos (Cavia porcellus) muestran frecuencias de 58 a $95 \%$ (Ruíz, 1961; Merino, 1991; Chauca, 1997; Gárate et al., 2008), las cuales demostrarían que este parásito es cosmopolita y de fácil adaptación a las condiciones medioambientales. Asimismo, se encontraron 
prevalencias de $P$. uncinata de 10 a $34 \%$ en bioterios (Casartelli et al., 2007; Coman et al., 2009) y de $40 \%$ en un establecimiento de venta de mascotas en Brasil (Magalhães et al., 2002). Estas prevalencias son inferiores a las del presente estudio, debido posiblemente a los cuidados y control sanitario existente en este tipo de establecimientos.

Flynn y Baker (2008) consideran a $P$. uncinata como un parásito no patógeno debido a la ausencia de lesiones en órganos; sin embargo, Coman et al. (2009), mediante cortes histopatólogicos observaron larvas en migración enteroparietal y lesiones en el ciego (éctasis de la submucosa capilar y tiflitis hemorrágica) y Conder et al. (1989) observaron aumento de eosinófilos en lavados traqueobronquiales en cuyes aparentemente sanos.

La afección por Trichuris spp produciría irritación mecánica, debido al daño que ocasiona en las paredes intestinales al alimentarse de exudado tisular y sangre (Quiroz, 2005). El $31 \%$ de prevalencia encontrado en el presente estudio es similar al 23\% reportado en cuyes silvestres (Tió, 1970), aunque bastante inferior al $62 \%$ reportado por Ruíz (1961) en cuyes domésticos. Para poder identificar la especie de Trichuris se requiere observar las características de la vaina de la espícula que está presente únicamente en los machos (Chandler, 1930), pero los especímenes encontrados en los cuyes fueron hembras en su totalidad; sin embargo, podría tratarse de Trichuris leporis, única especie reportada en conejos y roedores (Quiroz, 2005) y descrito por Sánchez (1984).

Capillaria hepatica ha sido reportado en el país afectando el hígado de cuyes silvestres (Dittmar, 2002); sin embargo, este órgano no fue evaluado en el presente estudio, y los especímenes encontrados estaban en el intestino delgado.

T. axei y T. colubriformis son considerados nematodos zoonóticos y presentan una prevalencia baja en personas que mantienen contacto estrecho con animales infectados y muestran pobres condiciones de salubridad (Wolfe, 1978; PAHO, 2003). El 2\% de prevalencia para T. colubriformis fue similar al $1 \%$ reportado por Ruíz (1961) aunque bastante inferior al 88\% de Trichostrongylus spp reportado para cuyes silvestres (Cavia aperea) en Brasil (Pereira, 2006). La patogenicidad de Trichostrongylus es baja y generalmente solo ocasiona infecciones asintomáticas dado que no se llega a observar lesiones en el intestino delgado; no obstante, cargas altas podrían producir diarrea acuosa y debilitante (Bowman, 2009).

No se encontró la presencia de céstodos en su forma adulta ni de metacéstodos; sin embargo, existen reportes en cuyes doméstico y silvestres (Tió, 1970; Bustamante, 1993; Pereira, 2006). No se pudo confirmar la presencia de Fasciola hepatica en los cuyes sacrificados al no haberse evaluado los hígados; sin embargo, este trematodo ha mostrado en la zona de estudio una prevalencia del $22 \%$ en ganado bovino (Manrique y Cuadros, 2002). No obstante, en la actualidad gran parte de la población de Caraz se encuentra incursionando en el cultivo de flores, e incluso la crianza de cuyes va migrando a otras áreas menos favorecidas para la agricultura.

\section{Conclusiones}

- Se encontró una prevalencia de $89 \%$ de nematodos gastrointestinales en cuyes de crianza familiar-comercial en el distrito de Caraz, Ancash.

- Se registraron cuatro especies de nematodos: Paraspidodera uncinata (83\%), Trichuris spp (31\%), Capillaria spp (18\%) y Trichostrongylus colubriformis (2\%).

- $\quad$ El $49 \%$ del parasitismo correspondió a monoparasitimo, el $35 \%$ a biparasitismo y el $5 \%$ a triparasitismo.

- No se encontró diferencia significativa entre el sexo de los cuyes y la presencia de parasitismo. 


\section{Agradecimientos}

Los autores agradecen la colaboración financiera del Consejo Superior de Investigaciones de la UNMSM para el desarrollo de esta investigación. Asimismo, al MV Miguel Siever Morales por las facilidades brindadas para la realización del presente estudio.

\section{Literatura Citada}

1. Botero D, Restrepo M. 2006. Parasitosis humanas. $4^{\circ}$ ed. Colombia: CIB. 506 p.

2. Bowman D. 2009. Georgis's parasitology for veterinarians. $9^{\text {th }}$ ed. China: Elsevier. $451 \mathrm{p}$.

3. Bustamante J. 1993. Producción de cuyes. Lima: Universidad Nacional Mayor de San Marcos. 259 p.

4. Casartelli L, Apolinario CC, Da Silva S, Reis SE, Caldas R. 2007. Endoparasitos em cobaias (Cavia porcellus) (Mammalia, Rodentia, Caviidae) provenientes de biotérios de criaçao e experimentaçao do municipio do Rio de Janeiro, Brasil. Rev Cienc Rural 37: 1380-1386.

5. Castro A, Guerrero O. 2006. Técnicas de diagnóstico parasitológico. $2^{\circ}$ ed. San José: Universidad de Costa Rica. 121 p.

6. Chandler A. 1930. Specific characters in the genus Trichuris, with a description of a new species, Trichuris tenuis from a camel. J Parasitology 16: 198-206.

7. Chauca L. 1997. Producción de cuyes (Cavia porcellus). Roma: FAO. 78 p.

8. Coman S, Bãcescu B, Coman T, Petrut P, Coman C, Vlase E. 2009. Aspects of the parasitary infestations of guinea pigs reared in intensive system. Sc Parasit 10(1-2): 97-100.

9. Conder G Richards I, Jen L, Marbury K, Oostveen J. 1989. Bronchoalveolar eosinophilia in guinea pigs harboring unapparent infection of Paraspidodera uncinata. J Parasitol 75: 144-146.
10. Cordero del Campillo M, RojoVázquez, Martínez A, Sánchez M, Hernández S, Navarrete I, et al. 2001. Parasitología veterinaria. Madrid: McGraw-Hill Interamericana. 968 p.

11. Daniel D. 1996. Bioestadística base para el análisis de las ciencias de la salud. $5^{\circ}$ ed. México: Limusa. 878 p.

12. Dean P, Stephen B. 2007. Pathology of laboratory rodents and rabbits. $3^{\text {rd }}$ ed. Iowa: Blackwell Publishing. 325 p.

13. Dittmar K. 2002. Arthropod and helminthes parasites of the wild guinea pig, Cavia aperea, from the Andes and the Cordillera in Peru, South America. J Parasitol 88: 409-411.

14. Florián A. 1999. Pérdidas de producción debido a enfermedades parasitarias. En: V Congreso Latinoamericano de Cuyicultura. Venezuela.

15. Flynn R, Baker D. 2008. Flynn's parasites of laboratory animals. $2^{\text {nd }} \mathrm{ed}$. USA: Wiley-Blackwell. 813 p.

16. Gárate I, Cueva B, Jiménez P, Portilla J, Uribe D, Villar J. 2008. Frecuencia e intensidad de infección por Paraspidodera uncinata en cobayos (Cavia sp.) sacrificados en Lima. En: XVII Reunión Científica del Instituto de Investigación de Ciencias Biológicas «Antonio Raimondi». Lima.

17. Griffiths H. 1971. Some common parasites of small laboratory animals. Lab Anim 5: 123-135.

18. Hakaro U, Cadral P. 1998. Manual para diagnóstico das helmintoses de ruminantes. $4^{\circ}$ ed. Brasil: Japan International Cooperation Agency. 120 p.

19. [INEI] Instituto Nacional de Estadística e Informática. 2012. [Internet], [7 febrero 2012]. Disponible en: www.inei.gob.pe

20. [INIA] Instituto de Investigación Agraria. 1994. Proyecto sistemas de producción de cuyes. Tomo II. Lima: INIA. 99 p.

21. Magalhães R, Corrêa D, MunizPereira L, Noronha D. 2002. Helminths of the guinea pig, Cavia 
porcellus (Linnaeus), in Brazil. Rev Bras Zool 19(Suppl 1): 261-269.

22. Manrique M, Cuadros C. 2002. Fasciolosis: Buscando estrategias de control. Perú: Akuarella. 126 p.

23. Merino S. 1991. Estudio preliminar de parásitos gastrointestinales en cuyes (Cavia porcellus) en el distrito de Cajamarca. Tesis de Médico Veterinario. Cajamarca: Univ Nacional de Cajamarca. 62 p.

24. [PAHO] Pan American Health Organization. 2003. Parasitoses. In: Zoonosis and communicable diseases common to man and animals. $3^{\circ}$ ed. $395 \mathrm{p}$.

25. Pereira C. 2006. Artrópodes e helmintos parasitos de Cavia aperea Exerleben, 1777 (Rodentia: Caviidae) no sul do Brasil. Tesis de Maestría. Brasil: Univ Federal de Pelotas. 63 p.

26. Quiroz H. 2005. Parasitología y enfermedades parasitarias de animales domésticos. México: Limusa. 876 p.

27. Ruiz M. 1961. Contribución al estudio de los parásitos gastrointestinales de $\mathrm{Ca}$ via cobaya en la provincia de Lima. Tesis de Médico Veterinario. Lima: Univ Nacional Mayor de San Marcos. 35 p.

28. Sánchez H. 1984. Prevalencia de helmintos gastrointestinales de cuyes (Cavia cobayo) en la provincia de Jauja. Tesis de Médico Veterinario y Zootecnia. Pasco: Univ Nacional Daniel Alcides Carrión. 53 p.
29. [SENAMHI] Servicio Nacional de Meteorología e Hidrología. 2009. En: Resumen técnico. Escenarios climáticos en la Cuenca del Río Santa para el año 2030. Lima: SENAMHI. 28 p.

30. Skrjabin K, Shikhobalova N, Shultz R. 1954. Essentials of nematology. Vol III. Trichostrongylids of animals and man. Moscow: Academy of Science. 350 p.

31. Soulsby EJL. 1987. Parasitología y enfermedades parasitarias en los animales domésticos. México: Interamérica. 823 p.

32. Thrusfield M. 1990. Epidemiología veterinaria. España: Acribia. 42 p.

33. Tió JA. 1970. Parásitos gastrointestinales en cobayos silvestres (Cavia aperea) de altura. Tesis de Médico Veterinario. Lima: Univ Nacional Mayor de San Marcos. 27 p.

34. Vicente JJ, De Olivera H, Correa D, Magalhães R. 1997. Nematóides do Brasil. Parte V: Nematóides de mamíferos. Rev Bras Zool 14: 1-452.

35. Wescott R. 1976. Helminth parasites. In: Wagner J, Manning P (eds). The biology of the guinea pig. New York: Academic Press. p 197-200.

36. Wisnivesky C. 2003. Ecología y epidemiología de las infecciones parasitarias. Costa Rica: Tecnológica de Costa Rica. 400 p.

37. Wolfe MS.1978. Oxyuris, trichostrongylus and trichuris. Rev Clin Gastroenterol 7: 201-217.

38. Yamaguti S. 1959. Sistema helmintum: The cestodes of vertebrates. New York: Interscience Publisher. 880 p. 